Acknowledgments. The authors would like to thank the Hospital Administration of Hong Kong Adventist Hospital and Gleneagles Hospital Hong Kong for their assistance throughout all aspects of the study.

Financial support. No financial support was provided relevant to this article.

Conflict of interest. B.J.C. is a consultant for AstraZeneca, GSK, Moderna, Pfizer, Roche and Sanofi Pasteur. The other authors report no other potential conflicts of interest.

\section{References}

1. Wong SYS, Kwok KO, Chan FKL. What can countries learn from Hong Kong's response to the COVID-10 pandemic? CMAJ 2020; 192: E511-E115.
2. Cheng VCC, Wong SC, Chen JHK, et al. Escalating infection control response to the rapidly evolving epidemiology of the coronavirus disease 2019 (COVID-19) due to SARSCoV-2 in Hong Kong. Infect Control Hosp Epidemiol 2020; 41: 493-498.

3. Cheng VCC, Siu GKH, Wong SC, et al. Complementation of contact tracing by mass testing for successful containment of beta COVID-19 variant (SARSCoV-2 VOC B.1.351) epidemic in Hong Kong. Lancet Reg Health W Pac 2021. doi: 10.1016/j.lanwpc.2021.100281.

4. Seto WH, Tsang D, Yung RM, et al. Effectiveness of droplets and contact precautions in prevention of nosocomial transmission of severe acute respiratory syndrome (SARS). Lancet 2003; 5: 1519-1520.

5. Lee WC, Ong CY. Overview of rapid mitigating strategies in Singapore during the COVID-19 pandemic. Public Health 2020; 185: 15-17.

\title{
Utilization of rapid antigen assays for detection of severe acute respiratory coronavirus virus 2 (SARS-CoV-2) in a low-incidence setting in emergency department triage: Does risk-stratification still matter?
}

\author{
Liang En Wee MRCP ${ }^{1}$ (1) , Edwin Philip Conceicao $\mathrm{BSC}^{2}$, Jean Xiang-Ying Sim MRCP ${ }^{1,2}$, \\ Indumathi Venkatachalam MRCP ${ }^{1,2}$, Paul Weng Wan MMed $^{3}$, Nur Diana Zakaria MCEM ${ }^{3}$, \\ Kenneth Boon-Kiat Tan MCEM ${ }^{3}$ and Limin Wijaya MRCP ${ }^{1}$ \\ ${ }^{1}$ Department of Infectious Diseases, Singapore General Hospital, Singapore, ${ }^{2}$ Department of Infection Prevention and Epidemiology, Singapore General Hospital, \\ Singapore and ${ }^{3}$ Department of Emergency Medicine, Singapore General Hospital, Singapore
}

To the Editor-We read with interest the article by Smith et $\mathrm{al}^{1}$ describing the use of rapid antigen detection (RAD) for severe acute respiratory coronavirus virus 2 (SARS-CoV-2) in guiding hospital admission triage. Given the rapid turnaround of pointof-care testing, various studies have utilized RAD tests at the point of entry into the healthcare system. ${ }^{1-4}$ However, most of these studies have occurred during periods of heightened transmission, with detection rates of $5 \%-21 \% .{ }^{1-4}$ RAD testing in clinical scenarios with lower incidence, such as asymptomatic individuals, may potentially result in low detection rates with high false-positive rates. $^{5}$ False-positive RAD tests have resulted in SARS-CoV-2negative patients being admitted to coronavirus disease 2019 (COVID-19) cohort wards, with subsequent nosocomial transmission. ${ }^{2}$ Conversely, given broad variations in potential infectivity among individuals with false-negative RAD tests, ${ }^{4}$ negative RAD tests need to be interpreted cautiously, especially in the context of significant contact history or clinical syndromes compatible with COVID-19. We describe our institution's experience with the implementation of RAD testing for SARS-CoV-2 to guide

\footnotetext{
Author for correspondence: Dr Wee Liang En, The Academia, 20 College Road, Singapore 169856. E-mail: ian.wee.l.e@singhealth.com.sg

Cite this article: Wee LE, et al. (2022). Utilization of rapid antigen assays for detection of severe acute respiratory coronavirus virus 2 (SARS-CoV-2) in a low-incidence setting in emergency department triage: Does risk-stratification still matter?. Infection Control \& Hospital Epidemiology, 43: 1974-1976, https://doi.org/10.1017/ice.2021.407
}

admission triage in a low-incidence setting. RAD testing was utilized in tandem with pre-existing triage strategies that stratified admissions according to epidemiological risk and clinical syndromes, ${ }^{6}$ which allowed a comparison of these approaches.

In Singapore, a Southeast Asian city-state, hospitals instituted admission triage strategies early on to segregate patients presenting with clinical syndromes compatible with COVID- $19 .{ }^{6}$ At our institution, the largest hospital campus in Singapore, patients with epidemiological risk were admitted directly to the isolation ward; patients without epidemiological risk who presented with clinical syndromes compatible with COVID-19 were isolated in modified cohort cubicles with reduced bed density in the "respiratory surveillance ward (RSW)" until SARS-CoV-2 was excluded by polymerase chain reaction (PCR) testing. ${ }^{6}$ During the first wave of COVID-19 from January to June 2020, these admission triage strategies were extremely successful in correctly placing suspected COVID- 19 cases. Although $\geq 1,500$ cases of COVID- 19 were managed in our institution, $<5 \%$ of cases were admitted outside isolation areas. ${ }^{7}$ However, with the emergence of more transmissible variant strains, a second wave of COVID-19 began at the end of April 2021, providing the impetus for universal screening of inpatients via PCR testing of respiratory samples on admission and every 7 days subsequently. On June 27, 2021, our institution began utilizing RAD testing to screen all admissions for SARS-CoV-2. Thereafter, patients with a positive RAD result were transferred 


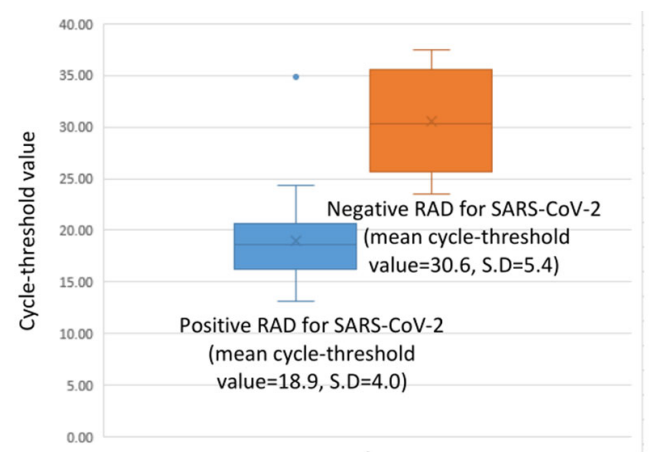

Data derived from all patients with concurrent RAD and PCR tests for SARS-CoV-2 from 24/5/21-8/8/21

Fig. 1. Cycle-threshold value on polymerase-chain-reaction (PCR) testing for SARS-CoV-2 E-gene of PCR-confirmed COVID-19 cases, stratified by rapid-antigendetection (RAD) result.

to isolation areas in the emergency department and then admitted to negative-pressure single rooms for confirmatory PCR testing. Patients with negative antigen tests were still risk-stratified for admission to isolation areas based on epidemiological risk and clinical syndromes. Our institution utilized the Veritor SARSCoV-2 antigen rapid test kit (Becton Dickinson, Franklin Lakes, NJ), with a positive percentage agreement of $\geq 80 \%$ and a negative percentage agreement of $99.5 \%$ compared to PCR testing. ${ }^{8}$

Over a 6-week study period from June 27, 2021, to August 8, 2021, 6,665 admissions were systematically tested for SARS-CoV2 with RAD tests and PCR. Moreover, 40 patients tested positive for SARS-CoV-2 by RAD; among them, 29 tested positive by PCR, with a mean cycle-threshold value for SARS-CoV-2 E-gene of 18.9 (Fig. 1). Two patients who tested negative for SARS-CoV2 by RAD subsequently tested positive by PCR; however, they were admitted initially to isolation due to epidemiological risk. The sensitivity of RAD was $93.6 \%$ (95\% CI, 78.6\%-99.2\%), with specificity of $99.8 \%$ ( $95 \%$ CI, $99.7 \%-99.9 \%)$. The positive predictive value of RAD was $72.5 \%$ (95\% CI, $59.2 \%-82.7 \%)$, and the negative predictive value was $99.9 \%$ (95\% CI, 99.8\%-99.9\%). Over the same period, 812 (12.2\%) of 6,665 admissions fulfilled epidemiological criteria and/or presented with a clinical syndrome compatible with COVID-19, and these patients were initially admitted to isolation areas while awaiting the results of PCR testing; 29 (3.6\%) of these 812 patients subsequently tested positive. The sensitivity of combined epidemiological and clinical criteria was $93.6 \%$ (95\% CI, 78.6\%-99.2\%), with a specificity of $88.2 \%$ (95\% CI, 87.4\%-88.9\%). The positive predictive value of epidemiological and clinical criteria was 3.6\% (95\% CI, 3.2\%-4.0\%), while the negative predictive value was $99.9 \%$ (95\% CI, 99.8\%-99.9\%). Two asymptomatic patients with no known epidemiological risk tested positive for SARS-CoV-2; 1 patient was admitted to isolation due to a positive RAD test. The sole case of COVID-19 admitted outside isolation occurred in an asymptomatic patient with no known epidemiological risk, for whom RAD testing was not performed due to previous nasal surgery.

Before the implementation of RAD testing, COVID-19 cases detected outside the isolation ward over a 6-month period (January-June 2020) spent an average of 16.5 hours $(\mathrm{N}=32$; $\mathrm{SD}, 9.76)$ from admission to isolation, with an average of 2.1 (SD, 3.25) inpatient close contacts identified for each index case. ${ }^{7,9}$ Of 68 inpatient close contacts identified, 1 patient subsequently tested positive within the incubation period. ${ }^{7,9}$ In contrast, after introduction of RAD testing, COVID-19 cases initially detected on RAD testing spent an average of 0.2 hours $(\mathrm{N}=29$; $\mathrm{SD}, 0.68)$ from admission to isolation (mean difference, -16.3 ; 95\% CI, -19.9 to $-12.7 ; P<.001$ ), with an average of 0.3 (SD, 0.84$)$ inpatient close contacts identified for each index case (mean difference, $-1.8 ; 95 \% \mathrm{CI},-3.5$ to $-0.06 ; P=.004$ ). Of 8 inpatient close contacts identified, none tested positive subsequently. No nosocomial COVID-19 clusters were detected among staff or by inpatient universal surveillance during the study period.

In conclusion, RAD testing for SARS-CoV-2 as part of an admission-triage strategy demonstrated high sensitivity and specificity, even in a low-incidence setting. Although a triage strategy based on epidemiological risk and clinical syndrome was resource-intensive, the potential for false-negative RAD tests and the possibility that patients with epidemiological risk could still be incubating at point of admission meant that RAD testing could not be used alone for isolation triage. The introduction of RAD testing substantially reduced potential exposure, in terms of both time to isolation and number of exposed inpatients. A robust admission triage strategy based on epidemiological risk and clinical syndrome, supported by an expansion of hospital isolation facilities $^{10}$ and RAD testing for earlier isolation of positive cases, successfully limited nosocomial exposures.

\section{Acknowledgments.}

Financial support. No financial support was provided relevant to this article.

Conflicts of interest. All authors report no conflicts of interest relevant to this article.

\section{References}

1. Smith RD, Johnson JK, Clay C, et al. Clinical evaluation of Sofia rapid antigen assay for detection of severe acute respiratory syndrome coronavirus 2 among emergency department to hospital admissions. Infect Control Hosp Epidemiol 2021. doi: 10.1017/ice.2021.281.

2. Van Honacker E, Van Vaerenbergh K, Boel A, De Beenhouwer H, LerouxRoels I, Cattoir L. Comparison of five SARS-CoV-2 rapid antigen detection tests in a hospital setting and performance of one antigen assay in routine practice: a useful tool to guide isolation precautions? J Hosp Infect 2021;114:144-152.

3. Leli C, Matteo LD, Gotta F, et al. Performance of a SARS CoV-2 antigen rapid immunoassay in patients admitted to the emergency department. Int J Infect Dis 2021. doi: 10.1016/j.ijid.2021.07.043.

4. Möckel M, Corman VM, Stegemann MS, et al. SARS-CoV-2 antigen rapid immunoassay for diagnosis of COVID-19 in the emergency department. Biomarkers 2021;26:213-220.

5. Kanji JN, Proctor DT, Stokes W, et al. Multicentre postimplementation assessment of the positive-predictive value of SARS-CoV-2 antigen-based point-of-care tests used for asymptomatic screening of continuing care staff. J Clin Microbiol 2021. doi: 10.1128/JCM.01411-21

6. Wee LE, Hsieh JYC, Phua GC, et al. Respiratory surveillance wards as a strategy to reduce nosocomial transmission of COVID-19 through early detection: the experience of a tertiary-care hospital in Singapore. Infect Control Hosp Epidemiol 2020;41:820-825.

7. Wee LE, Venkatachalam I, Sim XYJ, et al. Containment of COVID-19 and reduction in healthcare-associated respiratory viral infections through a multitiered infection control strategy. Infect Dis Health 2021; 26:123-131.

8. Young S, Taylor SN, Cammarata CL, et al. Clinical evaluation of BD Veritor SARS-CoV-2 point-of-care test performance compared to PCR-based testing and versus the Sofia 2 SARS antigen point-of-care test. J Clin Microbiol 2020;59(1):e02338-20. 
9. Wee LEI, Sim XYJ, Conceicao EP, et al. Containing COVID-19 outside the isolation ward: the impact of an infection control bundle on environmental contamination and transmission in a cohorted general ward. Am J Infect Control 2020;48:1056-1061.
10. Wee LE, Fan EMP, Heng R, et al. Construction of a container isolation ward: a rapidly scalable modular approach to expand isolation capacity during the coronavirus disease 2019 (COVID-19) pandemic. Infect Control Hosp Epidemiol 2020. doi: 10.1017/ice.2020.1222.

\title{
Frequent detection of severe acute respiratory syndrome coronavirus 2 (SARS-CoV-2) RNA on hands and skin of patients with coronavirus disease 2019 (COVID-19)
}

\author{
Sarah N. Redmond MD¹, Daniel F. Li MD²,2, Muhammed F. Haq MD², Lucas D. Jones BS ${ }^{3}$, Alexandria M. Nguyen MSHS , \\ Margaret Tiktin DNP4 ${ }^{4}$, Jennifer L. Cadnum BS ${ }^{3}$, Maria E. Navas MD ${ }^{5}$, Jessica Bingham RN ${ }^{6}$, Brigid M. Wilson $\mathrm{PhD}^{7}$ and \\ Curtis J. Donskey MD ${ }^{1,7}$ \\ ${ }^{1}$ Case Western Reserve University School of Medicine, Cleveland, Ohio, ${ }^{2}$ Research Service, Louis Stokes Cleveland VA Medical Center, Cleveland, Ohio, \\ ${ }^{3}$ Department of Molecular Biology and Microbiology, Case Western Reserve University School of Medicine, Cleveland, Ohio, ${ }^{4}$ Clinical Research Center, Louis \\ Stokes Cleveland VA Medical Center, Cleveland, Ohio, ${ }^{5}$ Pathology and Laboratory Medicine Services, Louis Stokes Cleveland VA Medical Center, Cleveland, Ohio, \\ ${ }^{6}$ Nursing Service, Louis Stokes Cleveland VA Medical Center, Cleveland, Ohio and ${ }^{7}$ Geriatric Research, Education, and Clinical Center, Louis Stokes Cleveland VA \\ Medical Center, Cleveland, Ohio
}

To the Editor-Hand hygiene is a core measure recommended for prevention of severe acute respiratory syndrome coronavirus 2 (SARS-CoV-2) transmission. ${ }^{1,2}$ Hand hygiene is needed to eliminate SARS-CoV-2 acquired on hands during contact with infected patients or contaminated surfaces and fomites, including personal protective equipment. ${ }^{1-3}$ In an in vitro model, SARS-CoV-2 inoculated onto human skin survived for 9 hours after inoculation versus 2 hours for influenza A. ${ }^{4}$ SARS-CoV-2 was rapidly inactivated on skin by $80 \%$ ethanol. $^{4}$

Hand hygiene may also be beneficial in reducing the burden of SARS-CoV-2 on the hands of individuals with coronavirus disease 2019 (COVID-19), and bathing may reduce SARS-CoV-2 on other skin sites. However, the burden of SARS-CoV-2 on hands and other skin sites of COVID-19 patients is unknown. Here, we tested the hypothesis that SARS-CoV-2 RNA is frequently present on the hands and the chest/abdomen of patients with COVID-19, particularly those with a high viral burden in samples collected from the anterior nares.

The study protocol was approved by the Cleveland VA Medical Center's Institutional Review Board. Between November 1, 2020, and April 26, 2021, a convenience sample of patients with symptomatic COVID-19 was enrolled. Patients were excluded if they had dementia or delirium, were admitted to the intensive care unit, and if $>3$ days had passed since their COVID-19 diagnosis.

Premoistened CLASSIQSwabs with universal transport medium (Copan Diagnostics, Murrieta, CA) were used to collect samples from the anterior nares, oropharynx, chest/abdomen $(10 \times 10-\mathrm{cm}$ surface area), and the entire surface area of both hands. Swabs were also used to sample environmental surfaces in patient rooms, including $5 \times 20-\mathrm{cm}$ areas of the bed rail and bedside table; outpatients sampled a frequently used chair and tabletop. A medical record

\footnotetext{
Author for correspondence: Curtis J. Donskey, E-mail: Curtis. Donskey@va.gov Cite this article: Redmond SN, et al. (2022). Frequent detection of severe acute respiratory syndrome coronavirus 2 (SARS-CoV-2) RNA on hands and skin of patients with coronavirus disease 2019 (COVID-19). Infection Control \& Hospital Epidemiology, 43: 1976-1977, https://doi.org/10.1017/ice.2021.403
}

review was conducted to obtain information on age, sex, medical conditions, and COVID-19 treatments.

A detailed description of the methods for RNA extraction and reverse transcriptase quantitative polymerase chain reaction (RT-qPCR) is included as Supplementary Material (online). The Fisher exact test was used to compare the proportions of COVID-19 patients with positive chest/abdomen or hand SARS-CoV-2 RNA for those with anterior nares cycle threshold $\left(C_{t}\right)$ values of $\leq 30$ or $>30$. The cycle threshold cutoff of 30 was chosen based on evidence that individuals with higher nasopharyngeal swab $C_{t}$ values are less likely to have a positive cultures or to transmit SARS-CoV-2 to contacts. ${ }^{5}$ Analyses were performed using $\mathrm{R}$ version 3.5.1 statistical software ( $\mathrm{R}$ Foundation for Statistical Computing, Vienna, Austria).

For the 16 study participants, the mean age was 60.8 years; $13(81.3 \%)$ were male; $9(56.3 \%)$ were receiving dexamethasone and supplemental oxygen at the time of enrollment; and 2 (12.5\%) were outpatients. The anterior nares RT-qPCR result was positive for SARS-CoV-2 in all participants with an average $C_{t}$ value of 29.2 (range, 18.2-40). Figure 1.A shows the percentage of other sites with positive RT-qPCR results for SARS-CoV-2 RNA, stratified by anterior nares $C_{t}$ value $\leq 30$ or $>30$. Of the 16 participants, the oropharynx, chest/abdomen, hand, and environmental samples were positive for SARS-CoV-2 RNA in 15 (93.8\%), 7 (43.8\%), 7 (43.8\%), and $3(18.8 \%)$ patients, respectively. All 7 patients with SARS-CoV-2 RNA on hands also had positive chest/abdomen samples. The percentage of chest/abdomen and hand samples that were positive was significantly higher in patients with anterior nares $\mathrm{C}_{\mathrm{t}}$ values of $\leq 30$ versus $>30$ : $6(75 \%)$ of 8 versus $1(12.5 \%)$ of $8(P=.04)$.

Figure $1 \mathrm{~B}$ shows a boxplot of the $\mathrm{C}_{t}$ values for SARS-CoV-2 RNA from all sites. Of the 7 patients with positive chest/abdomen RT-qPCR results for SARS-CoV-2, 1 patient (14.2\%) had a chest/ abdomen $C_{t}$ value $<30$ and 3 patients $(42.9 \%)$ had $C_{t}$ values between 30 and 35 . Of the 7 patients with positive hand RT-qPCR results for SARS-CoV-2, 3 patients (42.9\%) had hand $\mathrm{C}_{\mathrm{t}}$ values between 30 and 35 . Only $1(16.7 \%)$ of 6 positive 